\title{
A new interpretation of bulge test measurements using numerical simulation
}

\author{
Y. von Kaenel, J.-Ch. Giachetto, J. Stiegler, J.-M. Drezet, E. Blank \\ Ecole Polytechnique Fédérale de Lausanne, Département des Matériaux-LMPH, CH-1015 Lausanne, Switzerland
}

\begin{abstract}
Analysis of the deflection of a circular membrane under differential pressure (bulge test) is a well-known method of determining the elastic properties of thin films. However, analytical models always suffer from simplifying hypotheses. In this study we present a new approach, based on numerical modeling, to interpret pressure-deflection curves. By adjusting Young's modulus and Poisson's ratio in the simulation program, it is possible to reproduce the experimental curves faithfully. The method was successfully tested with two different materials (silicon and aluminium) with known elastic properties and was then used to determine biaxial Young's moduli of CVD diamond thin films for three different microstructures. The values of $E$ varied from 565 to $620 \mathrm{GPa}$ (assuming a Poisson ratio of 0.1). Grain boundaries are thought to be responsible for the relatively low values of Young's moduli. Uncertainties in $E$ are relatively large $(10 \%-15 \%)$ because the method is highly sensitive to experimental parameters such as thickness or membrane diameter and to the initial residual stress state which is known only approximately.
\end{abstract}

Keywords: Bulge test measurements; Numerical simulation; Young's modulus; CVD diamond; Thin films

\section{Introduction}

Recent developments in diamond CVD, allowing the fabrication of thin and thick films, have considerably enlarged the potential field of application of this exceptional material. The mechanical properties of diamond films, such as Young's modulus or fracture strength, are of prime importance for applications such as coatings for IR or optical windows. Different methods have been used to measure the elastic properties of diamond, for example Brillouin light scattering [1,2], ultrasonic methods $[3,4]$ and indentation techniques $[5,6]$. The biaxial modulus, which is the determinant parameter for most practical applications, can also be obtained by using the bulge test method which consists in measuring the central deflection of a membrane under differential pressure [7-9]. Because of the geometry of this test, only the biaxial modulus, which is isotropic for (100) planes and polycrystalline diamond and nearly isotropic for (110) planes [10], can be determined. This method, which also provides values of ultimate fracture strength, commonly suffers from simplifying assumptions necessary in interpreting pressure-deflection curves by analytical models, leading to large uncertainties. Internal residual stresses play an important role in the behavior of the membrane under pressure and must be taken into account for a correct interpretation of the bulge test measurements. The residual stress is generally considered to have two components [11]: thermal stress, which appears during cooling, and intrinsic stress whose origin is not yet fully understood. Defects, grain boundary formation and voids have been suggested as being responsible for the latter type of stress [9,11-13]. Young's moduli of CVD diamond reported in the literature for different types of layers, measured with different methods, range from 300 to $1100 \mathrm{GPa}$. Some parameters, such as the hydrogen content [5] or grain size (methane content) $[8,14]$, appear to affect Young's modulus.

In this study, the bulge test method was used to measure biaxial Young's moduli of three CVD diamond films with different morphologies (polycrystalline, fibertextured, and highly oriented and textured films) in order to investigate the effect of the microstructure on the elastic properties. The applied pressure was increased until bursting occurred to estimate the ultimate fracture strength and strain. A finite-element numerical analysis program was used to interpret the pressure-deflection curves, avoiding the simplifications required by analytical models and providing information on stress and strain in each point of the samples. 


\section{Experimental}

Three CVD diamond films were deposited onto silicon wafers of area approximately $1 \mathrm{~cm}^{2}$ in a microwave reactor at $850^{\circ} \mathrm{C}$. The polycrystalline sample was deposited on an ultrasonically pretreated (using diamond powder) wafer with $0.75 \% \mathrm{CH}_{4}$ in $\mathrm{H}_{2}$. The fiber-textured sample was pretreated in the same manner, but deposited with $1 \% \mathrm{CH}_{4}$ in $\mathrm{H}_{2}$. The highly oriented and textured specimen was also deposited with $1 \% \mathrm{CH}_{4}$ in $\mathrm{H}_{2}$ but on a wafer pretreated with a $-250 \mathrm{~V}$ bias. The deposition time was adapted in order to obtain a constant sample thickness of about $10 \mu \mathrm{m}$. The morphology of the samples is shown in Fig. 1.

After deposition, a hole of diameter $4 \mathrm{~mm}$ was etched in the center of the silicon substrate by acidic dissolution. The bulging of the diamond membrane upon removal of the substrate indicated the presence of a residual compressive stress or a stress gradient in the films (Fig. 2(a)), which had partially relaxed. The shape of the
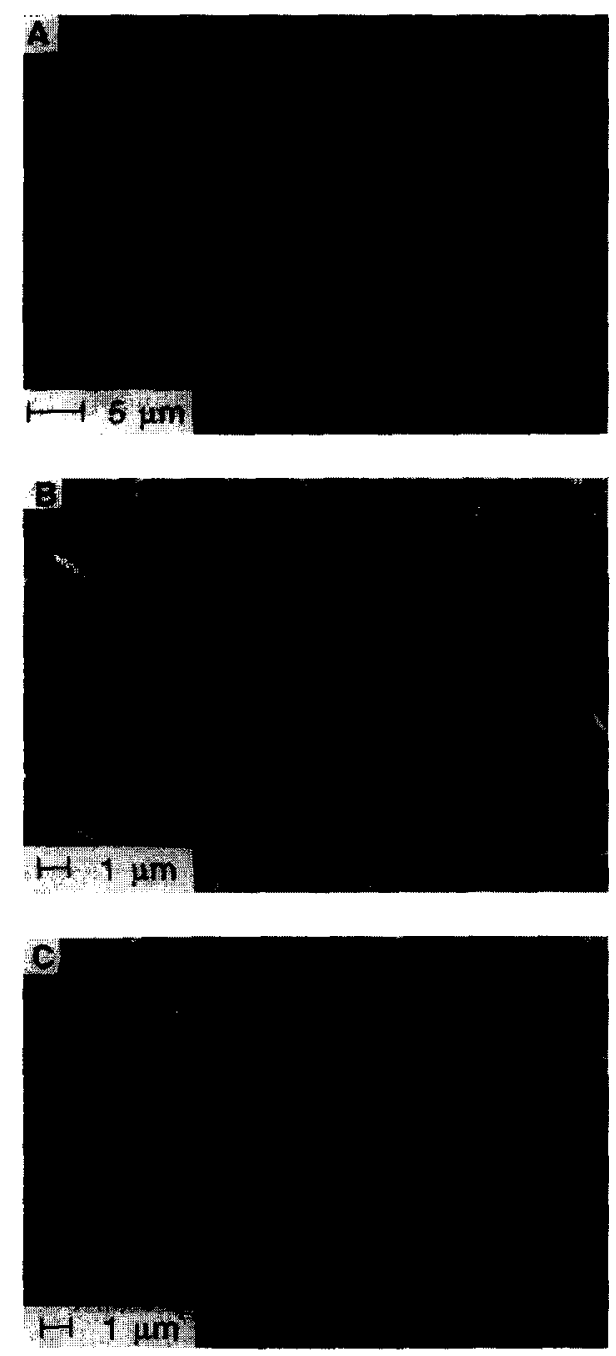

Fig. 1. SEM microstructures of the diamond films: (a) highly oriented and textured film; (b) fiber-textured film; (c) polycrystalline film.

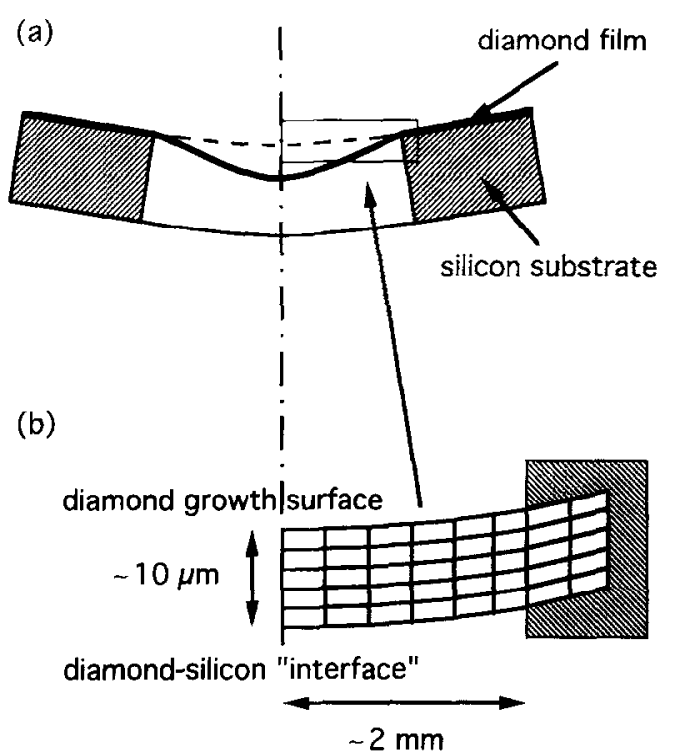

Fig. 2. (a) Schematic diagram of the shape of the diamond specimen showing bulging of the diamond membrane (thick black line) from its initial state (broken line), when the silicon substrate was removed. (b) The mesh used for the numerical simulation has the shape of the diamond membranes before the removal of the silicon substrate (dashed line in a)). The sample thickness was divided into five sublayers and the radius into 200 elements. Only half the film was considered because of the axial symmetry of the problem. The border of the film was fixed for the calculations.

deformed films was measured with a UBM laser profilometer with $10 \mathrm{~nm}$ resolution.

The diameter of the hole, which is a crucial parameter, was precisely measured under a microscope with a calibrated $X Y$ table with an accuracy of $\pm 50 \mu \mathrm{m}$. The film thickness was measured in the same manner, with an accuracy to $\pm 1 \mu \mathrm{m}$, on the side of film fragments after the burst test.

In order to validate the method, two test samples were fabricated with known materials. A hole of diameter $4 \mathrm{~mm}$ was made by spark machining in an aluminum plate $2 \mathrm{~mm}$ thick, leaving a membrane of thickness $55 \mu \mathrm{m}$. A hole of diameter $4.5 \mathrm{~mm}$ was etched with $\mathrm{KOH}$ in a silicon wafer $360 \mu \mathrm{m}$ thick, leaving a membrane of thickness $36.9 \mu \mathrm{m}$.

The bulge test apparatus consisted of a pressure cell and a He-Ne laser $(633 \mathrm{~nm})$ Michelson-type interferometer which was used to measure the membrane deflection with an accuracy to $0.2 \mu \mathrm{m}$ (Fig. 3(a)). The gas $\left(\mathrm{N}_{2}\right)$ inlet was controlled by a flowmeter and pressure was measured by a DIGIBAR mechanical gauge with a resolution of 10 mbar. A special sample holder, preventing the introduction of additional stresses due to assembly, was used (Fig. 3(b)). About 10 pressure loadingunloading cycles were necessary to obtain perfectly reproducible behavior with no measurable vertical displacement of the silicon substrate. During all these experiments, the pressure applied against the diamond 
(a)

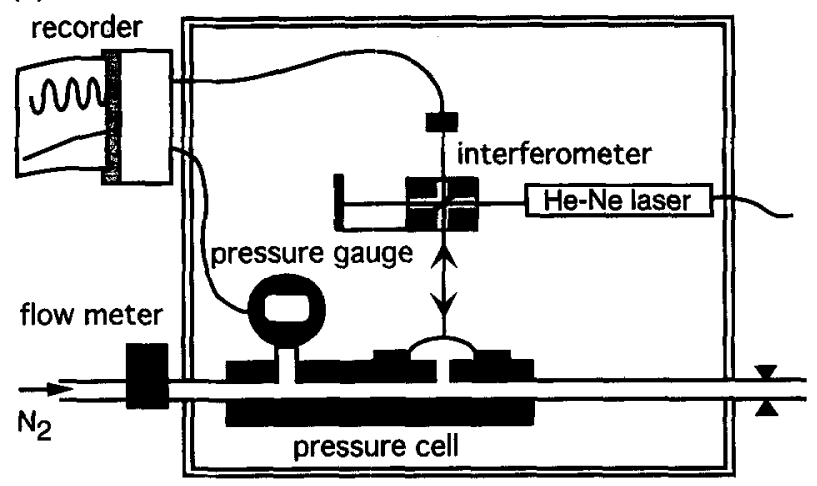

(b)

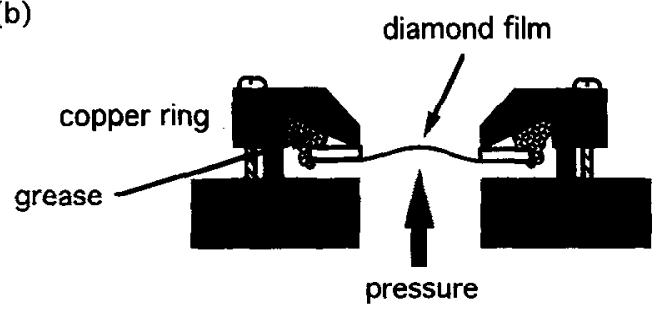

Fig. 3. (a) Diagram of the bulge test apparatus. Great care was taken to guarantee mechanical stability for the interferometric measurements: all metallic parts were water cooled, and the apparatus was mounted on a massive granite optical table and was protected from air flow with a covering box. (b) Detailed view of the sample holder. Specimens were fixed with viscous grease against a conical rigid copper ring. This prevented the introduction of additional stresses, which were observed with any other assembly (for instance O-rings). The maximum pressure available with this system was about $3.5 \times 10^{5} \mathrm{~Pa}$. At pressures above this value, the grease was extruded.

growth surface was recorded simultaneously with the intensity of the interferometer photodiode, yielding interference fringes.

The interpretation of the pressure-deflection curves was performed using the commercial finite-element package ABAQUS, assuming elastic deformation and axisymetric geometry (see mesh in Fig. 2(b)). The stress state of the film was calculated in the first step. As thermal stresses alone cannot explain the shape of the samples and as the origin of intrinsic stress is not yet understood, a stress source was introduced by artificially forcing the layers to expand. The expansion amplitude was adapted in such a way that the calculated shape of the sample corresponded to the experimental profiles (Fig. 4(a)). In the second step, pressure was applied to the bent films and Young's modulus $E$ was adjusted until the experimental pressure-deflection curve was reproduced precisely (Fig. 4(b)). As the program could not take the anisotropy of diamond into account, Poisson's ratio $v$ was fixed at 0.1 , but it was observed that varying $v$ between its extreme values of 0.07 and 0.2 , while keeping
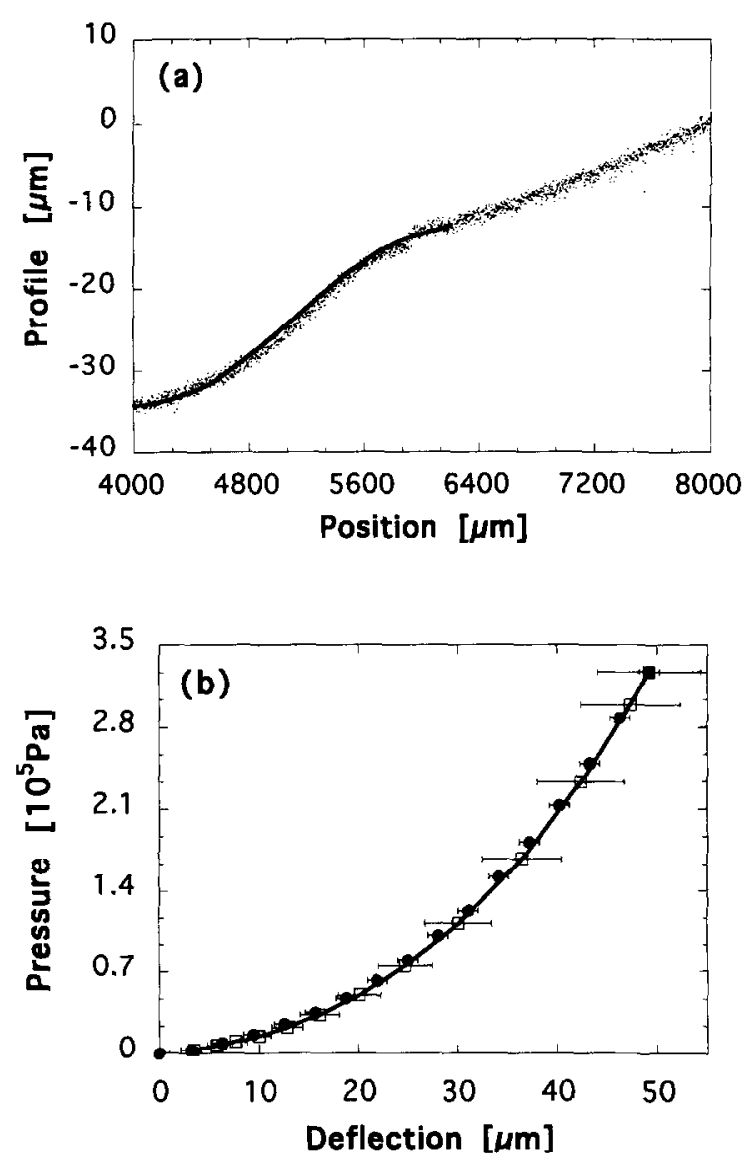

Fig. 4. Comparison between experiment and simulation: (a) experimental profile (dots) of the highly oriented and textured sample with the calculated shape after the simulation of stress formation $(-)$; (b) experimental (๑) and simulated ( $\square-$ ) pressure-deflection curves of the polycrystalline sample. Errors in the simulated points were estimated by replacing the values of the thickness and of the radius of the membrane in the program by their extreme values within their experimental uncertainty range, while retaining the same modulus.

the biaxial modulus $E^{*}=E /(1-v)$ constant, did not affect the calculated curves.

\section{Results and discussion}

The method was first tested with the aluminum and silicon test samples. As these two samples were stress free prior to the bulge test, only the second simulation step was used. Fig. 5 shows the good agreement obtained between the calculated and experimental pressuredeflection curves when using literature values for Young's modulus and Poisson's ratio for aluminum [15] and silicon [16] (see Table 1) in the program, thus confirming the validity of the method. The usual analytical models, which are based on either the bending of plates [17] (stress gradient across the film) or the bulging of membranes [7] (without bending and with uniform stress across the film), were also used for comparison. Plate theory provided a value of $E=73 \mathrm{GPa}$ for the aluminum 

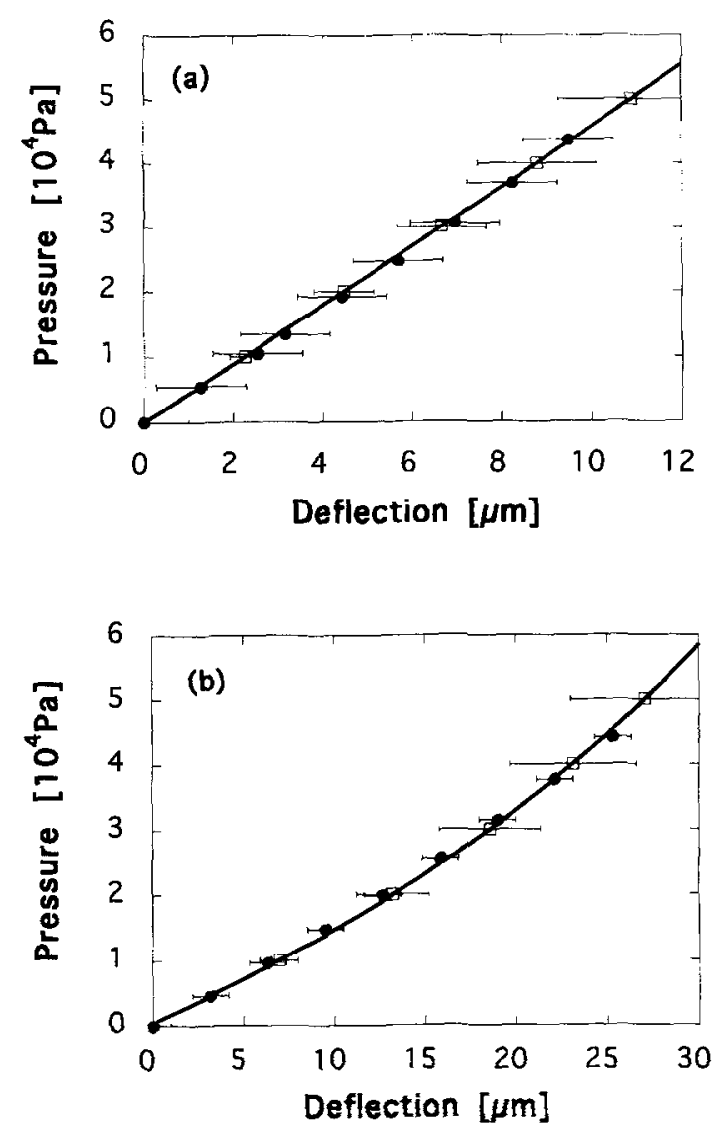

Fig. 5. Experimental pressure-deflection curves for (a) the $\mathrm{Al}$ and (b) the Si test samples superimposed on the curves simulated with tabulated values of Young's modulus and Poisson's ratio: experimental points; - extrapolation between the simulated points $(\square)$.

sample, but no satisfactory results were obtained for the silicon membrane, showing that the usual analytical models cannot properly describe films which have a behavior intermediate between that of a pure plate and a pure membrane (without bending), as is the case for the present silicon and thin diamond films. Numerical analysis revealed the presence of a stress gradient, which is also not allowed for by membrane analytical models, at the center of the membrane after the first calculation step, where uniform expansion over the film thickness was imposed to reproduce the experimental shape of the diamond layers. The growth surface had compressive stresses of $30-80 \mathrm{MPa}$ and interface tensile stresses of $50-100 \mathrm{MPa}$, depending on the sample. A stress difference of about $300 \mathrm{MPa}$ is still observed between the interface (at about $600 \mathrm{MPa}$ ) and the growth surface (at about $300 \mathrm{MPa}$ ) of the film after pressure loading (just before fracture), but the whole film is in tension, indicating that bending and stretching must be considered to interpret bulge test measurements. These values are difficult to compare with the literature, because only uniform stresses have been considered so far. As the evolution of the grain boundary density with the distance from the interface was known for the textured samples (calculated from the evolution of the grain size with film thickness) [18], an expansion gradient proportional to the grain boundary density was used in order to investigate whether grain boundaries could be at the origin of the stress state in the diamond films, as suggested in the literature [9,11-13]. Unfortunately, both expansion models fitted the experimental bent shapes and the pressure-deflection curves of the samples, and the same value of Young's modulus was obtained, thus preventing any distinction between the models. Modeling of the whole deposition process, accompanied by local stress measurements by the micro-Raman method for instance, would be necessary to reproduce the exact stress state of the diamond membranes. However, in the present numerical model, Young's modulus is no longer affected by the stress state as soon as the shape of the film is reproduced. Table 1 summarizes the data obtained for the three diamond films. The values of the biaxial modulus appear to be rather low compared with those for natural diamond [10].

Grain boundaries are known to contain noncrystalline carbon structures [19]. Therefore it would be of interest to determine whether grain boundaries are the origin of the relatively low Young's modulus of CVD diamond films. For this purpose, the film microstructure is assumed to be a composite built up of alternating crystalline and grain boundary elements which, with respect to the applied stress, are placed in a serial

Table 1

Summary of results

\begin{tabular}{llllllllll}
\hline Sample & $\begin{array}{l}\text { Radius } \\
(\mathrm{mm})\end{array}$ & $\begin{array}{l}\text { Thickness } \\
(\mu \mathrm{m})\end{array}$ & $\begin{array}{l}\text { Burst pressure } \\
\left(10^{5} \mathrm{~Pa}\right)\end{array}$ & $\begin{array}{l}E^{*}=E /(1-v) \\
(\mathrm{GPa})\end{array}$ & $\begin{array}{l}E^{*} \\
(\text { Ref. }[10])\end{array}$ & $\begin{array}{l}E \\
(\mathrm{GPa})\end{array}$ & $\begin{array}{l}\text { Fracture strength } \\
(\mathrm{MPa})\end{array}$ & $\begin{array}{l}\text { Fracture strain } \\
(\%)\end{array}$ \\
\hline Highly oriented & $1.99 \pm 0.05$ & $10.5 \pm 1$ & 3.15 & $667 \pm 60$ & $\begin{array}{l}1173 \\
(100) \text { plane }\end{array}$ & 600 & 0.1 & $630 \pm 60$ & 0.103 \\
Fiber-textured & $2.09 \pm 0.05$ & $8.7 \pm 1$ & 1.78 & $689 \pm 70$ & $\begin{array}{l}1173 \\
(100) \text { plane }\end{array}$ & 620 & 0.1 & $516 \pm 30$ & 0.08 \\
Polycrystalline & $2.04 \pm 0.05$ & $11.5 \pm 1$ & 3.27 & $628 \pm 60$ & $\begin{array}{l}1228 \\
\text { poly. }\end{array}$ & 565 & 0.1 & $591 \pm 30$ & 0.099 \\
Silicon & $2.25 \pm 0.1$ & $36.8 \pm 0.1$ & - & $180 \pm 5$ & - & $130 \pm 5$ & 0.28 & - & \\
Aluminum & $1.98 \pm 0.06$ & $55 \pm 1$ & - & $107.8 \pm 5$ & - & $70.6 \pm 5$ & 0.35 & - & - \\
\hline
\end{tabular}


sequence. This type of structure applies to the two textured samples for which the volume occupied by grain boundaries can be estimated, following the method outlined above and assuming a thickness of $10 \AA$ (two or three atomic layers) for the boundaries [19]. This volume represents roughly $0.5 \%$ of the total volume for both specimens. In this case, the Young's modulus of the diamond film can be expressed as

$E_{\mathrm{film}}=\frac{E_{\mathrm{gb}} E_{\text {diamond }}}{f_{\mathrm{gb}} E_{\text {diamond }}+\left(1-f_{\mathrm{gb}}\right) E_{\mathrm{gb}}}$

where $E_{\mathrm{gb}}$ is the modulus of the grain boundaries and $f_{\mathrm{gb}}$ is the volume fraction of the grain boundaries.

If Young's modulus for diamond is assumed to be $1050 \mathrm{GPa}$, a value of the order of $10 \mathrm{GPa}$ can be extracted for the modulus of the grain boundaries, which is similar to that found for glassy carbon [20]. This result suggests that grain boundaries are responsible for the weaker Young's modulus of CVD diamond films in comparison with the single-crystal material.

Ultimate fracture strengths (center of the membrane) are about five times lower than those for natural diamond, but similar data have been published previously [21]. The surface roughness may explain the difference between the samples: the highly oriented and textured sample has a lower roughness but a higher fracture strength, and vice versa for the textured specimen. The values for the polycrystalline film lie between these extremes. Other factors, such as the methanc concentration used during the deposition [14], have also been shown to influence the ultimate fracture strength. This is not observed here, perhaps because other parameters (pretreatment) have also been varied. The fracture strain is approximately $0.1 \%$, which corresponds to the fracture strain of hard ceramics such as alumina.

\section{Conclusions}

Numerical modeling of a membrane under differential pressure has been successfully used to interpret experimental pressure-deflection curves in terms of biaxial Young's moduli for three CVD diamond films with different morphologies, providing stress and strain values for each point of the sample. The usual analytical models were shown to be insufficient to describe bulging of thin diamond layers, whereas numerical analysis takes into account the non-spherical deflection of the films, any intermediate behavior between membrane and plate, and residual stresses. The biaxial modulus was observed to be independent of the way in which the residual stresses formation in the diamond films was modeled, provided that the bent shape of the sample prior to pressure testing was reproduced. An accurate stress determination would require the modeling of the whole deposition process. The rather low value for Young's modulus of CVD diamond films compared with single-crystal diamond was explained by the presence of the grain boundaries, assuming that they contain mainly amorphous phases with low modulus (about $10 \mathrm{GPa}$ ). The ultimate fracture strength of the "as-grown" layers, which is comparable with other published data, seems to depend on the surface roughness.

\section{Acknowledgment}

The authors gratefully acknowledge the Swiss Priority Program on Material research for financial support.

\section{References}

[1] M.H. Grimsditch and A.K. Ramdas, Phys. Rev. B, 11 (8) (1975) 3139.

[2] X. Jiang, J.V. Harzer, B. Hillebrands, Ch. Wild and P. Koidl, Appl. Phys. Lett., 59 (9) (1991) 1055.

[3] H.J. McSkimin and P. Andreatch, J. Appl. Phys., 43 (1972) 2944.

[4] L. Chandra and T.W. Clyne, J. Mater. Sci. Lett., 12 (1993) 191.

[5] C.V. Cooper and C.P. Beetz Jr., Surf. Coatings Technol., 47 (1991) 375 .

[6] S.J. Bull, P.R. Chalker, C. Johnston and C.V. Cooper, Diamond Relat. Mater., 4 (1994) 43.

[7] G.F. Cardinale and R.W. Tustison, J. Vac. Sci. Technol. A 9 (4) (1991) 2204.

[8] Y. Aikawa and K. Baba, Jpn. J. Appl. Phys., 32 (1993) 4680.

[9] H. Windischmann and G.F. Epps, Diamond Relat. Mater., 1 (1992) 656.

[10] C.A. Klein, Mater. Res. Bull., 27 (1992) 1407.

[11] L. Schäfer, X. Jiang and C.-P. Klages, Application of Diamond Films and Related Materials, Elsevier Science, Amsterdam, 1991, p. 121.

[12] D. Schwarzbach, R. Haubner and B. Lux, Diamond Relat. Mater., 3 (1994) 757.

[13] H. Windischmann and G.F. Epps, J. Appl. Phys., 69 (4) (1991) 2231.

[14] H. Windischmann, G.F. Epps and G.P. Cesar, Proc. 2nd Intern. Conf. New Diamond Science and Technology, Materials Research Society, Pittsburgh, PA, 1991, p. 767.

[15] A. Kelly and G.W. Groves, Crystallography and Crystal Defects, Longmans, London, 1970, p. 163.

[16] C.J. Smithells, Metal Reference Book (5th edn), Butterworths, London, p. 975.

[17] T.J. Valentine, A.J. Whitehead, R.S. Sussmann, C.J.H. Wort and G.A. Scarsbrook, Diamond Relat. Mater., 3 (1994) 1168.

[18] Y. von Kaenel, J. Stiegler, E. Blank, O. Chauvet, Ch. Hellwig and K. Plamann, Phys. Status Solidi (A), 154 (1996) in press.

[19] H. Eto, Y. Tamou, Y. Ohsawa and N. Kikuchi, Diamond Relat. Mater., 1 (1992) 373.

[20] J. Robertson, Solid State Chem., 2 I (1991) 199.

[21] G.F. Cardinale and C.J. Robinson, J. Mater. Res., 7 (6) (1992) 1432. 